\title{
ASCA X-ray Spectra of Quiescent and Flaring Emission from AB Doradus
}

\author{
S. M. White ${ }^{1}$, R. Pallavicini ${ }^{2}, J$. Lim $^{3}$ \\ 1 Department of Astronomy, University of Maryland, College Park MD 20742, USA \\ 2 Arcetri Astrophysical Observatory, Largo E. Fermi 5, I-50125 Florence, Italy \\ 3 Inst. for Astronomy and Astrophysics, Academica Sinica, Taipei
}

The Japanese satellite $A S C A$ carries $\mathrm{X}$-ray detectors which combine excellent sensitivity and high spectral resolution. We observed the young, rapidly-rotating $\mathrm{K}$ dwarf AB Doradus with $A S C A$ in November 1993. The star's X-ray flux was essentially steady for the first half of the observation, and then a series of flares occurred during the second half. The flares showed rise times of 30 minutes and decay times of several hours. The quiescent $X$-ray luminosity was $2 \times 10^{30}$ ergs $\mathrm{s}^{-1}(0.5-10 \mathrm{keV})$. At the flare peaks, $\mathrm{L}_{X}(>0.5 \mathrm{keV}) / \mathrm{L}_{\mathrm{bol}}=.002$.

$\mathrm{AB}$ Dor has a relatively high count rate in the $A S C A$ data and we can obtain high-quality spectra for different periods of the light curve. In Fig. 1 we present spectra corresponding to the period of quiescence, the rising phase and peak of each of the three flares combined, and the decay periods of each of the flares combined. The rise/peak curve (upper) has been multiplied by 2 for purposes of display. We have subtracted the quiescent spectrum from the flare spectra (i.e., used it as a background spectrum) for this display and subsequent analysis.

The detailed differences between these spectra display the importance of the high spectral resolution provided by $A S C A$. One important difference, not readily evident in this figure, is the $\mathrm{Fe} \mathrm{K}$ line complex at $6.4-6.7 \mathrm{keV}$. This line, a diagnostic of hot plasma, is strong in the rise/peak spectrum, moderate in the decay spectrum and weak in the quiescent spectrum. A time profile of the counts in this energy range shows little significant emission during the quiescent periods but strong emission at the flare peaks.

It is generally found that two-temperature, variable-abundance models fit ASCA data for active cool stars well. We have carried out such fits for the three separate spectra presented above (using the Mewe-Kaastra code, although we do not find major differences when using the Raymond-Smith code). We have only fit for the abundances of those elements whose abundances are reasonably well constrained by the spectrum, and we have forced the abundance to be the same in the hot and cool components. All three spectra show a cool component at $0.6 \mathrm{keV}$, but the emission measure of this component is much less in the two flare spectra than in the quiescent spectra, indicating (since we used the quiescent spectrum as a "background" for the flare spectra) that there was little significant enhancement in the cool component during the flares. The quiescent spectrum shows a hot coronal component at $1.7 \mathrm{keV}$ with about the same emission measure 
ASCA SISO data: AB Doradus (rise, decay, quiescent)

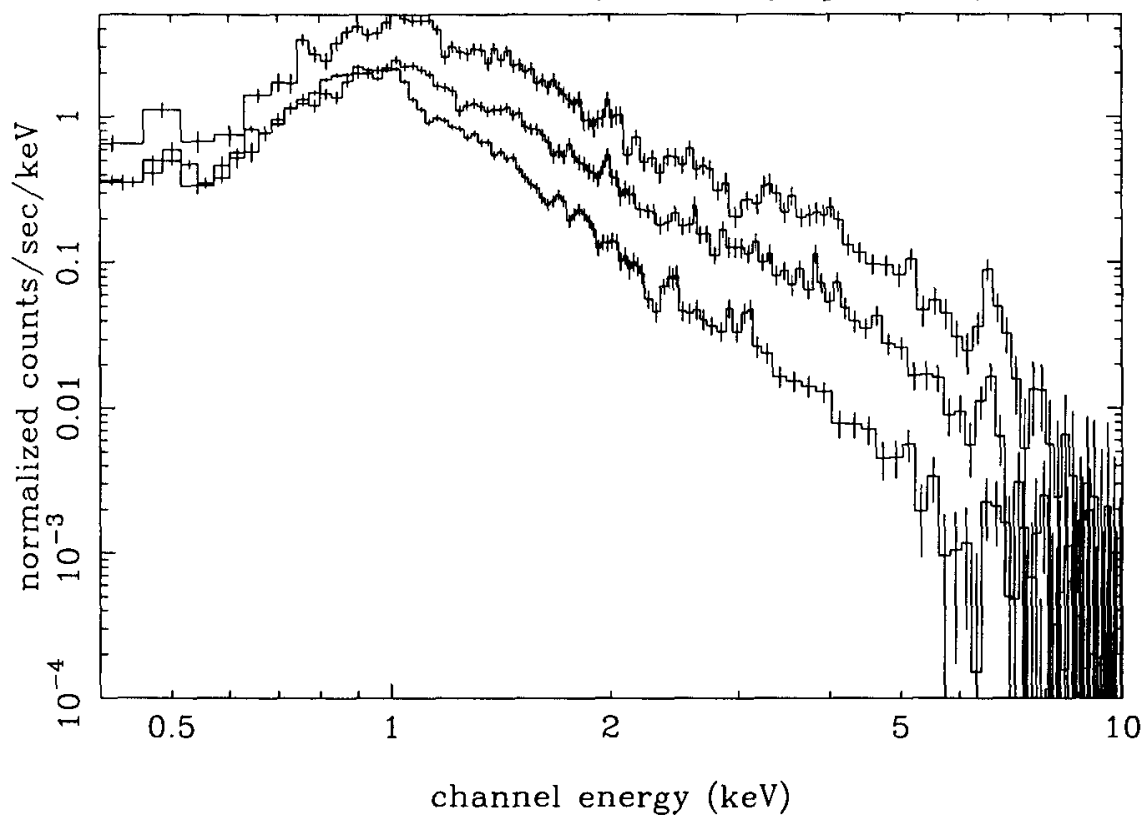

Fig. 1. $A S C A$ spectra of $\mathrm{AB}$ Dor in its quiescent state (lowest curve), during the rise and peak of three large flares (top curve, count rates multiplied by 2 for clearer display), and during the decay of the flares (middle curve).

as the cool component. The flare rise/peak spectrum has a strong hot component at $3.0 \mathrm{keV}$; the flare decay spectrum has a hot component with about the same emission measure as in the rise/peak spectrum but a cooler temperature, 2.4 $\mathrm{keV}$.

All three spectra show somewhat low iron abundances: 0.3 (relative to solar) in the quiescent spectrum, 0.8 in the flare rise/peak spectrum and 0.5 in the flare decay spectrum. The low abundance in the quiescent state is supported by aspects of the EUV spectrum obtained in a simultaneous EUVE observation (Rucinski et al. 1995). This is a puzzle because in the solar corona Fe, with its low first-ionization potential (FIP), is enhanced. All spectra also show low $\mathrm{N}$ abundances, a common feature of $A S C A$ spectra which may be partly instrumental (a prominent $\mathrm{N}$ line lies at the low-energy cutoff of the SIS detectors).

\section{References}

Rucinski S., Mewe R., Kaastra J., Vilhu O., White S. M., 1995, ApJ (submitted) 